\title{
COMPARISON BETWEEN USUAL AND VECTOR TIME DERIVATIVES
}

\author{
ÓSCAR LÓPEZ-POUSO \\ Univ. Santiago de Compostela, Dep. Mat. Aplicada, Fac. Matemáticas, Campus Sur s/n, \\ 15782 Santiago de Compostela (A Coruña, SPAIN) \\ e-mail: oscarlp@usc.es
}

(Received 5 February, 2002; accepted 17 April, 2002)

\begin{abstract}
We prove two comparison theorems between the time derivative of a real function $u(x, t)$ such that $u(\cdot, t)$ belongs to $\mathrm{L}^{1}(\Omega)$ for all $t$, and the time derivative of the vector function $\hat{u}(t)=u(\cdot, t)$.
\end{abstract}

2000 Mathematics Subject Classification. 47D03, 47H20.

1. Introduction. Let us consider a model problem consisting in a system of partial differential equations, with the characteristic of being evolutive. This problem may perhaps be studied with the aid of well-known theories such as the Hille-Yosida or the Crandall-Liggett generation theorems [1-4]. In this case the system of partial differential equations has to be written as an abstract Cauchy problem, abstract in the sense that the unknown $u(x, t)$ is turned into a function $\hat{u}(t)$ with values in a Banach or Hilbert space of functions, and such that

$$
\hat{u}(t)(x)=u(x, t) .
$$

To illustrate the readers, the heat equation

$$
\begin{aligned}
& \frac{\partial u}{\partial t}-\Delta u=0 \quad \text { in } \quad \Omega \times[0, T], \\
& u(x, t)=0 \quad \text { on } \quad \partial \Omega \times[0, T], \\
& u(x, 0)=u_{0}(x) \text { in } \Omega \text {, }
\end{aligned}
$$

turns into the abstract Cauchy problem

$$
\begin{aligned}
\frac{d \hat{u}}{d t}+A \hat{u} & =0, \\
\hat{u}(0) & =u_{0},
\end{aligned}
$$

where $A \hat{u}(t)=-\Delta[\hat{u}(t)]$ and the apparently missing boundary condition is hidden in the domain of $A$; that is to say, $A$ is defined on a set of functions all of which satisfy the boundary condition (3) (see [2]).

It is true that, when using semigroup theory (linear or nonlinear) to study existence of solution, the time derivative is not understood in the classical sense, and the "semigroup" solution (mild solution; also $\mathrm{C}^{0}$-solution) does not need to be differentiable. Thus, in general one does not have to be concerned with the time derivatives. However, one could think that, when $u$ is differentiable with respect to time, $\partial u / \partial t$ 
and $d \hat{u} / d t$ are the same object. What we try in this paper is to bring the reader's attention on the fact that the existence of $\partial u / \partial t$, without further hypotheses, does not imply the existence of $d \hat{u} / d t$.

The author is aware that the results contained in this paper are known to some experts working in PDEs; however, it is the author's opinion that the paper is still valuable, since there is no precise reference where they can be found.

2. Results. We prove the comparison theorems assuming that the Banach space of functions is $\mathrm{L}^{1}(\Omega)$, since this work is inspired by [3]. Hence let us assume that

$$
u: \Omega \times[0, T] \rightarrow \mathbb{R}
$$

(where $\Omega$ is an open subset of $\mathbb{R}^{N}$ ) is such that

$$
u(\cdot, t) \in \mathrm{L}^{1}(\Omega) \quad \forall t \in[0, T],
$$

and let us define, for $t \in[0, T]$,

$$
\hat{u}(t)=u(\cdot, t)
$$

We use the notation "s-lim" to mean strong limit; to be precise, if $\psi_{n}$ is a sequence in $\mathrm{L}^{1}(\Omega)$,

$$
\mathrm{s}-\lim _{n \rightarrow \infty} \psi_{n}=\psi
$$

means, first, $\psi \in \mathrm{L}^{1}(\Omega)$, and, second,

$$
\lim _{n \rightarrow \infty}\left\|\psi_{n}-\psi\right\|_{L^{1}(\Omega)}=0 .
$$

Analogously, differentiability of $\hat{u}$ means strong differentiability.

Whenever we mention derivatives at 0 or at $T$, it is implicitly assumed that we are meaning "right" or "left" derivatives, respectively.

THEOREM 2.1. Let us suppose that

$$
\hat{u}:[0, T] \rightarrow \mathrm{L}^{1}(\Omega)
$$

is differentiable on $[0, T]$, and that, for all $t \in[0, T]$ and for a.e. $x \in \Omega$,

$$
\frac{\partial u}{\partial t}(x, t) \text { exists and is in } \mathbb{R} \text {. }
$$

Then, for all $t \in[0, T]$,

$$
\frac{d \hat{u}}{d t}(t)=\frac{\partial u}{\partial t}(\cdot, t) \quad \text { in } \quad \mathrm{L}^{1}(\Omega)
$$

Proof. Set $t \in(0, T)$. Strong differentiability of $\hat{u}$ means that the strong limit

$$
\frac{d \hat{u}}{d t}(t)=\operatorname{sim}_{h \rightarrow 0} \frac{\hat{u}(t+h)-\hat{u}(t)}{h}
$$


exists in $\mathrm{L}^{1}(\Omega)$. Let us define, for $n \in \mathbb{N}$ large enough,

$$
\psi_{n}=n[\hat{u}(t+1 / n)-\hat{u}(t)] .
$$

Then $\psi_{n} \in \mathrm{L}^{1}(\Omega)$ and

$$
\mathrm{s}-\lim _{n \rightarrow \infty} \psi_{n}=\frac{d \hat{u}}{d t}(t)
$$

which implies, for a certain subsequence $\psi_{n_{k}}$ of $\psi_{n}$,

$$
\lim _{k \rightarrow \infty} \psi_{n_{k}}(x)=\left[\frac{d \hat{u}}{d t}(t)\right](x) \text { a.e. in } \Omega .
$$

On the other hand, according to the definition of $\psi_{n_{k}}$ and $\hat{u}$,

$$
\lim _{k \rightarrow \infty} \psi_{n_{k}}(x)=\frac{\partial u}{\partial t}(x, t) \text { for a.e. } x \text { in } \Omega,
$$

and now we conclude the result from (18) and (19) and the uniqueness of limit.

The proof for $t=0$ and $t=T$ can be performed analogously, taking into account right or left derivatives.

FirST COUNTEREXAMPLE. We notice that it is possible to find a function

$$
u: \Omega \times[0, T] \rightarrow \mathbb{R}
$$

such that

- $u(\cdot, t) \in \mathrm{L}^{1}(\Omega)$ for all $t \in[0, T]$,

- $\partial u / \partial t(x, t)$ exists and is in $\mathbb{R}$ for all $(x, t) \in \Omega \times[0, T]$,

and such that $\hat{u}$ is not strongly differentiable at some $t \in[0, T]$. For example, let us consider

$$
\Omega=(0,1) \subset \mathbb{R}, \quad[0, T]=[0,1],
$$

and

$$
u(x, t)=\ln (x+t) .
$$

Then

$$
\frac{\partial u}{\partial t}(x, t)=\frac{1}{x+t} \quad \forall(x, t) \in(0,1) \times[0,1],
$$

and

$$
\frac{d \hat{u}}{d t}(0)
$$

does not exist in $\mathrm{L}^{1}(0,1)$, since in that case, according to Theorem 2.1,

$$
\frac{d \hat{u}}{d t}(0)(x)=\frac{\partial u}{\partial t}(x, 0)=\frac{1}{x},
$$

which does not belong to $\mathrm{L}^{1}(0,1)$. 
THEOREM 2.2. Let us suppose that, for all $t \in[0, T]$ and for a.e. $x \in \Omega$,

$$
\frac{\partial u}{\partial t}(x, t) \text { exists and is in } \mathbb{R},
$$

and that

$$
\sup _{t \in(0, T)}\left|\frac{\partial u}{\partial t}(x, t)\right| \leq F(x) \quad \text { for a.e. } x \in \Omega, \text { with } F \in \mathrm{L}^{1}(\Omega) .
$$

Then, for all $t \in[0, T]$,

$$
\frac{d \hat{u}}{d t}(t) \text { exists and is in } \mathrm{L}^{1}(\Omega)
$$

and

$$
\frac{d \hat{u}}{d t}(t)=\frac{\partial u}{\partial t}(\cdot, t) \text { in } \mathrm{L}^{1}(\Omega)
$$

Proof. Set $t \in[0, T]$, and define

$$
\psi_{h}=\frac{\hat{u}(t+h)-\hat{u}(t)}{h}
$$

for $h$ small enough. It is clear that

$$
\lim _{h \rightarrow 0} \psi_{h}(x)=\frac{\partial u}{\partial t}(x, t) \quad \text { a.e. in } \Omega \text {. }
$$

Now we must prove that

$$
\mathrm{s}-\lim _{h \rightarrow 0} \psi_{h}=\frac{\partial u}{\partial t}(\cdot, t)
$$

to conclude the result. Notice that

$$
\psi_{h}(x)=\frac{\partial u}{\partial t}\left(x, t^{\star}\right)
$$

for some $t^{\star}$ strictly placed between $t$ and $t+h$ (thus $t^{\star}$ is in the open interval $(0, T)$ ), and consequently

$$
\left|\psi_{h}(x)\right|=\left|\frac{\partial u}{\partial t}\left(x, t^{\star}\right)\right| \leq \sup _{t \in(0, T)}\left|\frac{\partial u}{\partial t}(x, t)\right| \leq F(x) \quad \text { a.e. in } \Omega,
$$

which implies (31), in virtue of (30) and the dominated convergence theorem.

REMARK 2.1. Theorem 2.2 implies that $d \hat{u} / d t(t)$ exists in $\mathrm{L}^{1}(\Omega)$ (and it is equal to $\partial u / \partial t(\cdot, t))$ provided that

$$
\begin{aligned}
& \Omega \text { is bounded, and } \\
& \frac{\partial u}{\partial t} \text { (exists in } \mathbb{R} \text { and it) is bounded in } \Omega \times[0, T] .
\end{aligned}
$$


A careful reading of the proof of Theorem 2.2 shows that the hypothesis (26)

[H1] $\alpha(x)=\sup _{t \in(0, T)}\left|\frac{\partial u}{\partial t}(x, t)\right| \leq F(x)$ for a.e. $x \in \Omega$, with $F \in \mathrm{L}^{1}(\Omega)$,

can be replaced either by

[H2] For each $\bar{t} \in[0, T]$, there exist $\delta=\delta(\bar{t})>0$ and $G_{\bar{t}, \delta} \in \mathrm{L}^{1}(\Omega)$ such that

$$
\beta_{\bar{t}, \delta}(x)=\sup _{t \in V(\bar{t}, \delta)}\left|\frac{\partial u}{\partial t}(x, t)\right| \leq G_{\bar{t}, \delta}(x) \quad \text { for a.e. } x \in \Omega,
$$

where $V(\bar{t}, \delta)=(\bar{t}-\delta, \bar{t}+\delta) \cap(0, T)$,

or by

[H3] For each compact set $K \subset[0, T]$, there exists $H_{K} \in \mathrm{L}^{1}(\Omega)$ such that

$$
\gamma_{K}(x)=\sup _{t \in K}\left|\frac{\partial u}{\partial t}(x, t)\right| \leq H_{K}(x) \quad \text { for a.e. } x \in \Omega,
$$

Indeed, there seems to be some relationship between [H1] and the local hypotheses [H2] and [H3]. The reader can prove the following proposition.

Proposition 2.1. Let us suppose that, for all $t \in[0, T]$ and for a.e. $x \in \Omega$,

$$
\frac{\partial u}{\partial t}(x, t) \text { exists and is in } \mathbb{R} .
$$

Then [H1], [H2] and [H3] are equivalent.

SECOND COUNTEREXAMPLE. We notice that it is possible to find a function

$$
u: \Omega \times[0, T] \rightarrow \mathbb{R}
$$

such that

- $u(\cdot, t) \in \mathrm{L}^{1}(\Omega)$ for all $t \in[0, T]$,

- $\partial u / \partial t(x, t)$ exists and is in $\mathbb{R}$ for all $(x, t) \in \Omega \times[0, T]$,

- $\partial u / \partial t(\cdot, t) \in \mathrm{L}^{1}(\Omega)$ for all $t \in[0, T]$,

and such that $\hat{u}$ is not strongly differentiable at some $t \in[0, T]$. For example, let us consider

$$
\begin{aligned}
\Omega=(0,1) & \subset \mathbb{R}, \quad[0, T]=[0,1], \quad R=\Omega \times[0, T], \\
R_{1} & =\{(x, t) \in R: t \geq 2 x\}, \\
R_{2} & =\{(x, t) \in R: x / 2 \leq t \leq 2 x\} \\
R_{3} & =\{(x, t) \in R: t \leq x / 2\}
\end{aligned}
$$

and

$$
u(x, t)= \begin{cases}-\frac{4 x}{t}+\frac{15}{4} & \text { if }(x, t) \in R_{1}, \\ \frac{t}{x}-\frac{1}{4} & \text { if }(x, t) \in R_{2}, \\ \frac{t^{2}}{x^{2}} & \text { if }(x, t) \in R_{3} .\end{cases}
$$


Then it is easily checked that $u$ is differentiable with respect to $t$, and that

$$
\frac{\partial u}{\partial t}(x, t)= \begin{cases}\frac{4 x}{t^{2}} & \text { if }(x, t) \in R_{1}, \\ \frac{1}{x} & \text { if }(x, t) \in R_{2}, \\ \frac{2 t}{x^{2}} & \text { if }(x, t) \in R_{3} .\end{cases}
$$

The reader can verify that $u(\cdot, t)$ and $\partial u / \partial t(\cdot, t)$ belong to $\mathrm{L}^{1}(\Omega)$ for all $t \in[0,1]$. However, $d \hat{u} / d t(0)$ does not exist in $\mathrm{L}^{1}(\Omega)$, since (recall Theorem 2.1)

$$
\mathrm{s}_{h \rightarrow 0} \frac{\hat{u}(h)-\hat{u}(0)}{h} \neq \frac{\partial u}{\partial t}(\cdot, 0) \equiv 0 .
$$

In order to check (43), it suffices to see that

$$
\lim _{n \rightarrow \infty} \int_{0}^{1} n\left(\int_{0}^{1 / n} \frac{\partial u}{\partial t}(x, s) d s\right) d x \neq \int_{0}^{1} \frac{\partial u}{\partial t}(x, 0) d x=0 .
$$

If we call $A_{n}=\{(x, t) \in R: 0 \leq t \leq 1 / n\}$, we have for all $n$

$$
\begin{aligned}
\int_{0}^{1} n\left(\int_{0}^{1 / n} \frac{\partial u}{\partial t}(x, s) d s\right) d x & =n \int_{A_{n}} \frac{\partial u}{\partial t}(x, s) d s d x \\
\geq n \int_{A_{n} \cap R_{2}} \frac{\partial u}{\partial t}(x, s) d s d x & =n \int_{A_{n} \cap R_{2}} \frac{1}{x} d s d x=\ln 4
\end{aligned}
$$

which implies (44).

Regarding this counterexample, we note that hypothesis (26) is not satisfied, and consequently Theorem 2.2 cannot be applied.

ACKNOWLEDGEMENTS. The author wishes to express his gratitude to the referee for advice which has made the paper more accurate.

\section{REFERENCES}

1. Ph. Bénilan, M. G. Crandall and A. Pazy, Nonlinear evolution governed by accretive operators (Springer-Verlag, to appear).

2. H. Brézis, Analyse fonctionnelle: théorie et applications (Masson, 1983).

3. O. López-Pouso, Trace theorem and existence in radiation, Adv. Math. Sci. Appl. 10 (2000) no. 2, 757-773.

4. R. E. Showalter, Monotone operators in Banach space and nonlinear partial differential equations, Mathematical Surveys and Monographs, Vol. 49 (AMS, 1997). 